\title{
Optical image processing to determine the optical properties of cellular membranes
}

\author{
M. L. Calvo, M. Chevalier, and C. Carreras
}

\begin{abstract}
In this paper we present a theoretical model for the transmission function of a biological cell. Three different cases are considered: real transmission function, pure phase object, general case, where both real and complex terms are present in the transmission function. In all cases we studied the response in the Fourier plane. Comparison of the proposed models leads us to conclude that the most correct treatment would come from the general case, where it was found that the membrane contribution predominates in the total diffracted amplitude distribution.
\end{abstract}

\section{Introduction}

For many years scattering techniques have been a useful tool to determine physical parameters of small particles, ${ }^{1,2}$ e.g., a biological cell. If the call is assumed to be formed by two concentric spheres, the scattering pattern can be given by the Lorentz-Mie theory.

Even if the scattering techniques give information about the object in three dimensions, a biological cell can also be considered a bidimensional object in which the different regions contributing to the cellular morphology do not have the same optical properties and, hence, different transmission coefficients, ${ }^{3}$ that is, the cell can be treated as an inhomogeneous object. On the other hand, it is well known that it is possible to represent the optical behavior of a biological cell in a simple way by assuming it formed by two concentric circles, an inner one corresponding to the nucleus and an outer one representing the cytoplasm. ${ }^{4}$ Thus, a transmission function with circular symmetry is defined for the cell, where only two transmission factors are considered. Anyway, important chemical reactions take place in the external zone of the cell formed by the membrane, in the sense that for a healthy cell the optical properties of the membrane could in fact be different from those corresponding to an unhealthy one. Thus, to include addi-

M. L. Calvo is with Universidad Complutense, Departamento de Optica, Madrid 3, Spain; M. Chevalier is with Universidad Complutense, Catedra de Fisica Medica, Madrid 3, Spain; and C. Carreras is with UNED, Departamento de Electricidad y Magnetismo, Madrid 3, Spain.

Received 31 May 1983.

0003-6935/84/020324-06\$02.00/0.

(C) 1984 Optical Society of America. tional information corresponding to the membrane, a new theoretical model is considered in which we have included a gradual variation in the chemical composition of the membrane. It is then a fair approximation to associate it with a gradient for the refractive index, varying slowly from the cytoplasm-membrane boundary to the external surface of the membrane. The simplest function representing the transmission factor for the membrane would then be a parabolic function.

\section{Mathematical Model for the Transmission Function of a Biological Cell}

\section{A. General Case}

The most general treatment for a biological cell leads to the introduction of a transmission function $t(r)$, where both the amplitude term representing the attenuation coefficient and the exponential term representing the complex phase have to be considered. However, it is also possible to make several assumptions in order to study the cell as a pure amplitude and a pure phase object. In all these cases the intensity responses in the Fourier plane have been calculated by applying a Bessel-Fourier transform to the associated $t(r)$; consequently it is possible to obtain a range of values for the spatial frequencies. ${ }^{5}$ Accordingly

$$
t(r)=A(r) \exp [i \Phi(r)]
$$

The attenuation coefficient $A(r)$ is defined (see Fig. 1) as

$$
A(r)= \begin{cases}t_{N} & 0 \leq r \leq d_{N} / 2 \\ t_{C} & d_{N} / 2 \leq r \leq d_{C} / 2, \\ t_{M}(r) & d_{C} / 2 \leq r \leq d_{T} / 2\end{cases}
$$

where $t_{N}$ and $t_{C}$ are constant factors associated with the 


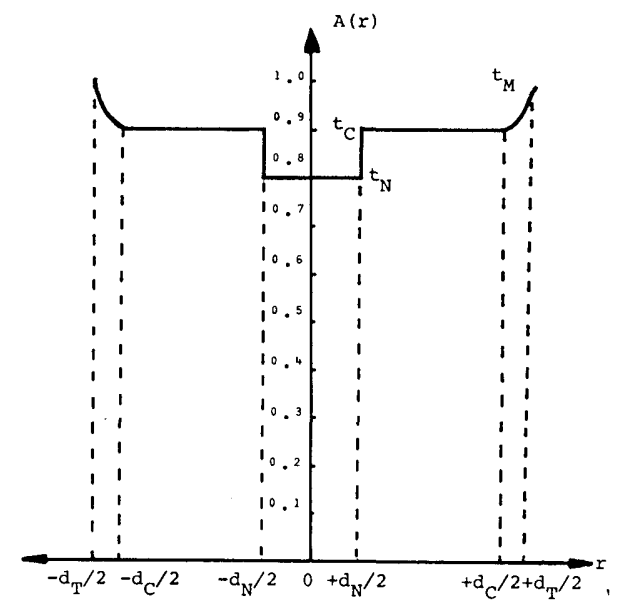

Fig. 1. Theoretical model for the real transmission function of a biological cell.

- nucleus and the cytoplasm, respectively, and $t_{M}$ is the factor corresponding to the membrane represented as

$$
t_{M}(r)=\frac{t_{M}-t_{C}}{\left(d_{T}-d_{C}\right)^{2}}\left(2 r-d_{C}\right)^{2}+t_{C} ;
$$

here $d_{N}, d_{C}$, and $d_{T}$ are the diameters corresponding to the nucleus, the cytoplasm, and the total cell, respectively.

The phase term $\Phi(r)$ is expressed as dictated by geometrical optics as

$$
\Phi(r)=k \sum_{1}^{3} \Delta_{i},
$$

and it contains the contribution of the different profiles of the cell as well as the variation in the refractive index of the corresponding zones of the cell. The factor $k$ which appears in Eq. (4) is the wave number. Then $\Phi(r)$ is defined as

$$
\Phi(r)= \begin{cases}\Phi_{N} & 0 \leq r \leq d_{N} / 2, \\ \Phi_{C} & d_{N} / 2 \leq r \leq d_{C} / 2, \\ \Phi_{M} & d_{C} / 2 \leq r \leq d_{T} / 2 .\end{cases}
$$

As a first approximation, $\Phi_{N}, \Phi_{C}$, and $\Phi_{M}$ are constant phase factors associated with the nucleus, the cytoplasm, and the membrane, respectively.

The transmission function defined in Eq. (1) becomes

$$
t(r)= \begin{cases}t_{N} \exp \left(i \Phi_{N}\right) & 0 \leq r \leq d_{N} / 2 \\ t_{C} \exp \left(i \Phi_{C}\right) & d_{N} / 2 \leq r \leq d_{C} / 2 \\ t_{M}(r) \exp \left(i \Phi_{M}\right) & d_{C} / 2 \leq r \leq d_{T} / 2\end{cases}
$$

Let us consider an incoming monochromatic plane wave propagating from the remote past. This plane wave illuminates normally the object plane where the cell is placed. The complex amplitude distribution in the Fourier plane is given in terms of the Bessel-Fourier transform of the transmission function $t(r)$ defined in Eq. (6). Thus

$$
\Psi(R)=\frac{1}{i \lambda z} \exp (i k z) \exp \left(i k R^{2} / 2 z\right)\{B[t(r)]\},
$$

where $r$ is the radial coordinate in the object plane and $R$ is the radial coordinate in the Fourier plane. By substituting Eq. (6) into Eq. (7) and operating conveniently we obtain $\Psi(R)$ as the sum of three separate terms:

$$
\Psi(R)=\frac{\exp (i k z)}{i \lambda z} \exp \left(i k R^{2} / 2 z\right)\left[\sum_{1}^{3} A_{i}(\rho)\right]_{\rho=R / \lambda z},
$$

where

$$
\begin{aligned}
A_{1}(\rho)= & {\left[t_{N} \exp \left(i \Phi_{N}\right)-t_{C} \exp \left(i \Phi_{C}\right)\right] \frac{d_{N}^{2}}{4} \frac{J_{1}\left(\pi \rho d_{N}\right)}{\pi \rho d_{N}} } \\
A_{2}(\rho)= & t_{C}\left[\exp \left(i \Phi_{C}\right)-\exp \left(i \Phi_{M}\right] \frac{d_{C}^{2}}{4} \frac{J_{1}\left(\pi \rho d_{C}\right)}{\pi \rho d_{C}}\right. \\
& +\exp \left(i \Phi_{M}\right) \frac{t_{M}-t_{C}}{\left(d_{T}-d_{C}\right)^{2}} 0.08334 d_{C}^{4} \frac{J_{1}\left(\pi \rho d_{C}\right)}{\pi \rho d_{C}} \\
& -\exp \left(i \Phi_{M}\right) \frac{t_{M}-t_{C}}{\left(d_{T}-d_{C}\right)^{2}}\left[\frac{d_{C}^{3}}{2 \pi \rho} \frac{J_{2}\left(\pi \rho d_{C}\right)}{\pi \rho d_{C}}\right. \\
& \left.-0.05 d_{C}^{4} \frac{J_{3}\left(\pi \rho d_{C}\right)}{\pi \rho d_{C}}\right], \\
A_{3}(\rho)= & t_{C} \exp \left(i \Phi_{M}\right) \frac{d_{T}^{2}}{4} \frac{J_{1}\left(\pi \rho d_{T}\right)}{\pi \rho d_{T}} \\
& +\exp \left(i \Phi_{M}\right) \frac{d_{T}^{2}}{4} \frac{J_{1}\left(\pi \rho d_{T}\right)}{\pi \rho d_{T}} \frac{t_{M}-t_{C}}{\left(d_{T}-d_{C}\right)^{2}} \\
& \times\left(d_{C}^{2}-1.3334 d_{C} d_{T}\right)+\exp \left(i \Phi_{M}\right) \frac{t_{M}-t_{C}}{\left(d_{T}-d_{C}\right)^{2}} \\
& \cdot\left[\frac{d_{T}^{3}}{2 \pi \rho} \frac{J_{2}\left(\pi \rho d_{T}\right)}{\pi \rho d_{T}}-\frac{d_{T}^{4}}{4} \frac{J_{3}\left(\pi \rho d_{T}\right)}{\pi \rho d_{T}}\right. \\
& \left.+0.2 d_{T}^{3} d_{C} \frac{J_{3}\left(\pi \rho d_{T}\right)}{\pi \rho d_{T}}\right] \cdot
\end{aligned}
$$

The $A_{1}(\rho)$ contribution corresponds to the diffraction pattern due to the nucleus. It is given in terms of an Airy function whose argument depends on the radius of the nucleus; it is affected by a transmission factor given as the difference between the transmission factors of the nucleus and the cytoplasm, respectively. All the cytoplasm spatial frequencies are included in the $A_{2}(\rho)$ contribution. The first term on the right-hand side of Eq. (10) represents the pure diffraction pattern due to the isolated cytoplasm. The factor multiplying it comes as a consequence of the discontinuity in the phase terms associated with the cytoplasm and the membrane, respectively. The other two terms appear since the attenuation coefficient is continuous at the cytoplasmmembrane boundary. Finally, in the $A_{3}(\rho)$ contribution all the membrane spatial frequencies are present. As in the two above-mentioned equations, the first term is due to the pure membrane diffraction. The second term on the right-hand side of Eq. (11) is also the result of the continuity in the attenuation coefficients. The second-and third-order Bessel functions appearing in the last term come from the parabolic behavior of the membrane attenuation coefficient. The total intensity response in the Fourier plane is directly obtained by squaring Eq. (8).

All the Bessel functions with orders higher than three have been neglected in the above expressions. It is a good approximation as their contributions to the exact formula are very small. 


\section{B. Real Transmission Function}

Let us assume that the profile effect is negligible. then $\Phi(r)=0$ in Eq. (1) and the real transmission function is defined by Eq. (6) where the complex terms now disappear. The complex amplitude distribution in the Fourier plane is still expressed as in Eq. (8) but several differences appear in the $A_{i}$ terms. Thus

$$
\begin{aligned}
A_{1}(\rho)= & \left(t_{N}-t_{C}\right) \frac{d_{N}^{2}}{4} \frac{J_{1}\left(\pi \rho d_{N}\right)}{\pi \rho d_{N}}, \\
A_{2}(\rho)= & \frac{t_{M}-t_{C}}{\left(d_{T}-d_{C}\right)^{2}} 0.08334 d_{C} \frac{J_{1}\left(\pi \rho d_{C}\right)}{\pi \rho d_{C}}-\frac{\left(t_{M}-t_{C}\right)}{\left(d_{T}-d_{C}\right)^{2}} \\
& \cdot\left[\frac{d_{C}^{3}}{2 \pi \rho} \frac{J_{2}\left(\pi \rho d_{C}\right)}{\pi \rho d_{C}}-0.05 d_{C}^{4} \frac{J_{3}\left(\pi \rho d_{C}\right)}{\pi \rho d_{C}}\right] \\
A_{3}(\rho)= & t_{C} \frac{d_{T}^{2}}{4} \frac{J_{1}\left(\pi \rho d_{T}\right)}{\pi \rho d_{T}} \\
+ & \frac{d_{T}^{2}}{4} \frac{t_{M}-t_{C}}{\left(d_{T}-d_{C}\right)^{2}} \frac{J_{1}\left(\pi \rho d_{T}\right)}{\pi \rho d_{T}}\left(d_{C}^{2}-1.3334 d_{C} d_{T}\right) \\
+ & \frac{t_{M}-t_{C}}{\left(d_{T}-d_{C}\right)^{2}}\left[\frac{d_{T}^{3}}{2 \pi \rho} \frac{J_{2}\left(\pi \rho d_{T}\right)}{\pi \rho d_{T}}-\frac{d_{T}^{4}}{4} \frac{J_{3}\left(\pi \rho d_{T}\right)}{\pi \rho d_{T}}\right. \\
+ & \left.0.2 d_{T}^{3} d_{C} \frac{J_{3}\left(\pi \rho d_{T}\right)}{\pi \rho d_{T}}\right] .
\end{aligned}
$$

The physical meaning of Eqs. (12)-(14) remains the same as in the general case explained before. Anyway, the most important difference appears in Eq. (13), where the term for the phase discontinuities between the cytoplasm and the membrane has disappeared.

\section{Pure Phase Transmission Function}

A second particular case of Eq. (1) would come by assuming the attenuation coefficients equal to unity in the whole cell. Thus we regard the latter as a pure object phase. Then, in Eq. (6) only the complex terms remain. Note that in this case the squared modulus of the transmission function is equal to unity. Physically that means that the cell is supposed to be transparent. Operating in the same way as in the preceding section the complex amplitude distribution given in Eq. (8) is expressed in terms of the new $A_{i}$ functions as

$$
\begin{aligned}
& A_{1}(\rho)=\left[\exp \left(i \Phi_{N}\right)-\exp \left(i \Phi_{C}\right)\right] \frac{d_{N}^{2}}{4} \frac{J_{1}\left(\pi \rho d_{N}\right)}{\pi \rho d_{N}} \\
& A_{2}(\rho)=\left[\exp \left(i \Phi_{C}\right)-\exp \left(i \Phi_{M}\right)\right] \frac{d_{C}^{2}}{4} \frac{J_{1}\left(\pi \rho d_{C}\right)}{\pi \rho d_{C}} \\
& A_{3}(\rho)=\exp \left(i \Phi_{M}\right) \frac{d_{T}^{2}}{4} \frac{J_{1}\left(\pi \rho d_{T}\right)}{\pi \rho d_{T}}
\end{aligned}
$$

From Eq. (15) we deduce that the pure diffraction pattern due to the isolated nucleus would come from the differences between the phase terms associated with the nucleus and the cytoplasm, respectively. The same interpretation remains for the cytoplasm. Equations (16) and (17) are simpler than in the real transmission function case. Basically, two reasons exist for this result: continuity in the attenuation coefficient has been removed; the parabolic behavior for the membrane has been lost. On the other hand, in Eq. (17) the factor multiplying the Airy function depends only on $\Phi_{M}$, as the diffraction takes place in the external boundary separating the cell from the surrounding medium; (note that a vanishing phase is associated with the latter).

\section{Numerical Results}

To understand the variation of the total intensity distribution of a diffracted field by a biological cell with the proposed mathematical model, some numerical estimations have been carried out. For that purpose, the following physical parameters have been assumed:

Incoming radiation wavelengths: $\lambda=632.8 \mathrm{~nm}$.

Nucleus attenuation coefficient: $t_{N}=0.8$.

Cytoplasm attenuation coefficient: $t_{C}=0.9$.

Membrane attenuation coefficient: $t_{M}=1.0$.

Nucleus total diameter: $d_{N}=2 \times 10^{-5} \mathrm{~m}$.

Cytoplasm total diameter: $d_{C}=7.9 \times 10^{-5} \mathrm{~m}$.

Cell total diameter: $d_{T}=8.0 \times 10^{-5} \mathrm{~m}$.

Equivalent focal length (corresponding to a planachromatic microscope objective with $100 \times$ magnification): $f=0.148 \mathrm{~m}$.

\section{A. Numerical Results for the General Case}

Using these physical parameters, the three separate contributions to the total diffracted amplitude distribution given in Eqs. (9)-(11) have been computed. The results obtained are displayed in Figs. 2-4. The total diffracted amplitude is shown in Fig. 5, the total diffracted intensity distribution is shown in Fig. 6 . The most important contribution comes from the parabolic behavior of the membrane attenuation coefficient. In all cases the different values for the phases $\Phi$ have been assumed, varying from $\Phi=0$ to $\Phi=\pi / 2$ and from $\Phi=$ $\pi$ to $\Phi=3 \pi / 2$. The maximum contributions with physical meaning for the nucleus, the cytoplasm, and the membrane have been found for the following phase values: $\Phi_{N}=\pi / 8, \Phi_{C}=0.01$, and $\Phi_{M}=9 \pi / 8$.

We note that the three partial contributions, as well as the total one, oscillate strongly due to the exponential term $\exp \left(i k R^{2} / 2 z\right)$. Anyway, it is easy to see that the distributions of the pulses in the three partial contributions do not coincide with one another, and their corresponding profiles appear to be quite different. This seems to be an interesting result and could be an important source of information in the Fourier plane response. Note that this effect is enhanced as the diffraction pattern is very extended in the Fourier plane. The total diffracted intensity distribution displayed in Fig. 6 presents a high central maximum and two weaker secondary maxima as was expected from the circular symmetry of the object.

\section{B. Numerical Results for the Real Transmission Function}

In Fig. 7 the diffracted amplitude distribution due to the nucleus by itself is displayed. Figures 8 and 9 correspond to the contributions of the cytoplasm and the membrane, respectively. Important differences were not found when comparing with the general case, although there is a variation in the spatial frequency values where the pulses are defined. Still, the maximum contribution to the total amplitude distribution 


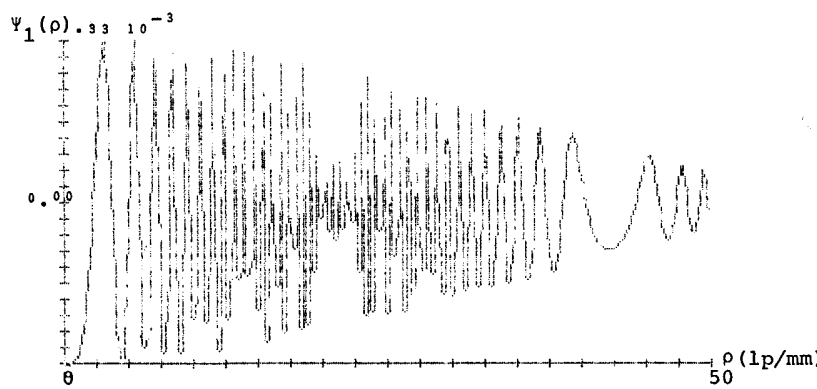

Fig. 2. Nucleus contribution to the total diffracted complex amplitude distribution: general case $\left(\Phi_{N}^{\prime}=\pi / 8, \Phi_{C}=0.01, \Phi_{M}=\right.$ $9 \pi / 8)$.

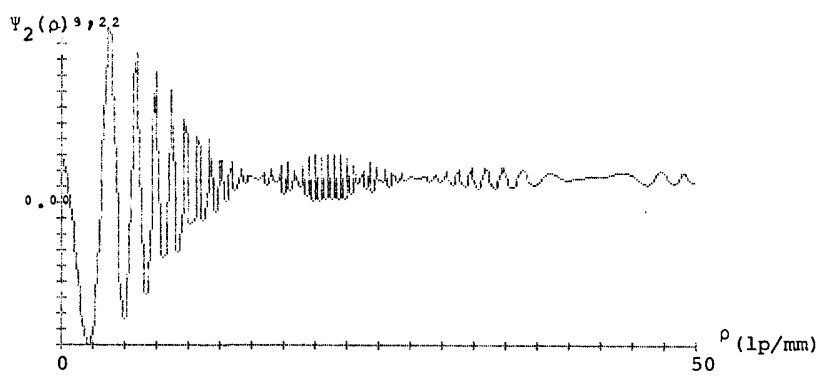

Fig. 3. Cytoplasm contribution to the total diffracted complex amplitude distribution: general case $\left(\Phi_{N}=\pi / 8, \Phi_{C}=0.01, \Phi_{M}=\right.$ $9 \pi / 8)$.

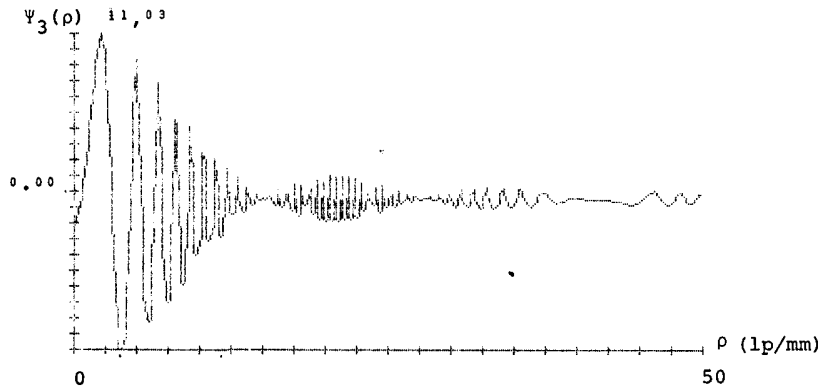

Fig. 4. Cellular membrane contribution to the total diffracted complex amplitude distribution: general case $\left(\Phi_{N}=\pi / 8, \Phi_{C}=0.01\right.$, $\left.\Phi_{M}=9 \pi / 8\right)$.

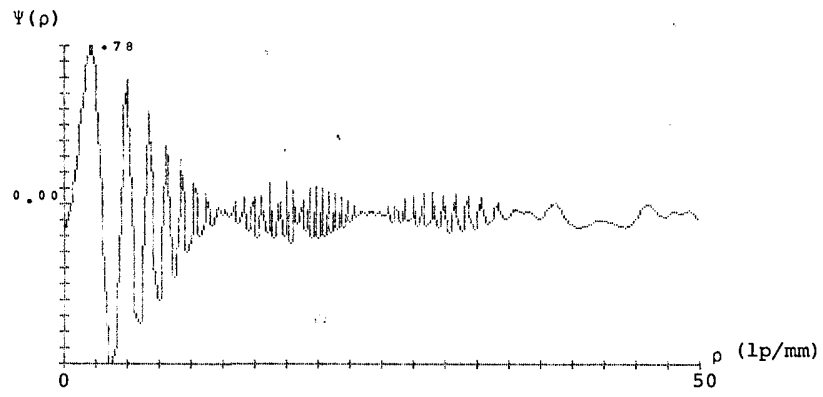

Fig. 5. Total diffracted amplitude distribution for the general case $\left(\Phi_{N}=\pi / 8, \Phi_{C}=0.01, \Phi_{M}=9 \pi / 8\right)$.

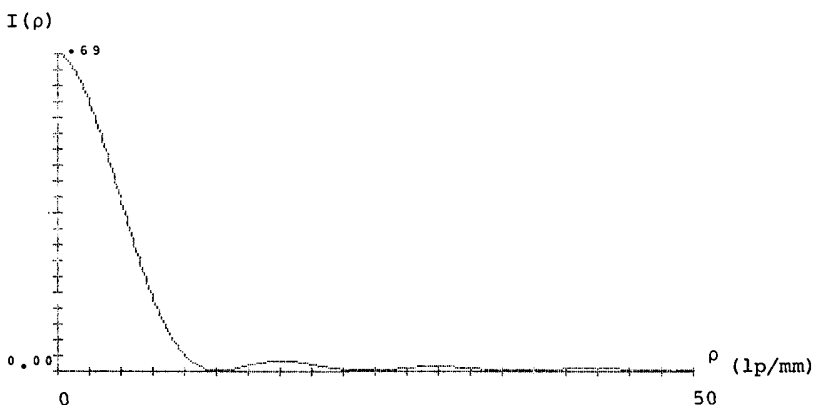

Fig. 6. Total diffracted intensity distribution for the general case $\left(\Phi_{N}=\pi / 8, \Phi_{C}=0.01, \Phi_{M}=9 \pi / 8\right)$.

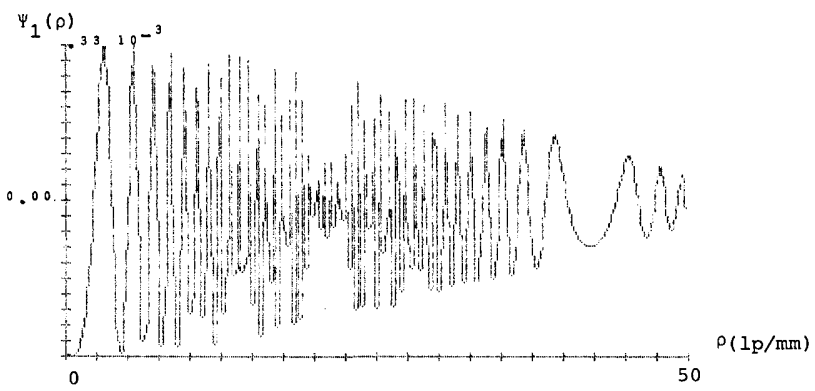

Fig. 7. Nucleus contribution to the total diffracted complex amplitude distribution for the real transmission function.

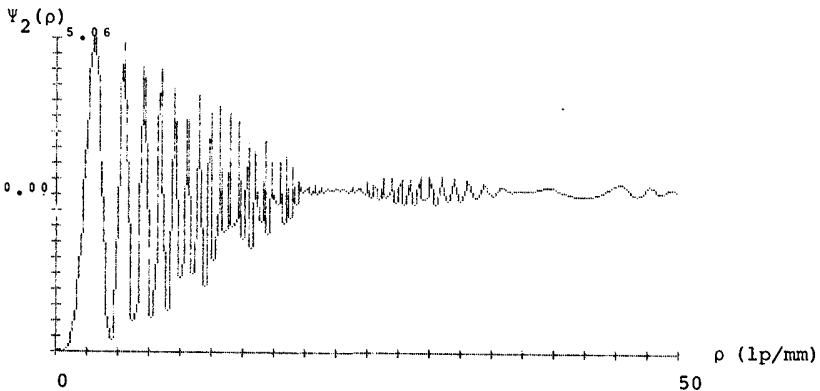

Fig. 8. Cytoplasm contribution to the total diffracted complex amplitude distribution for the real transmission function.

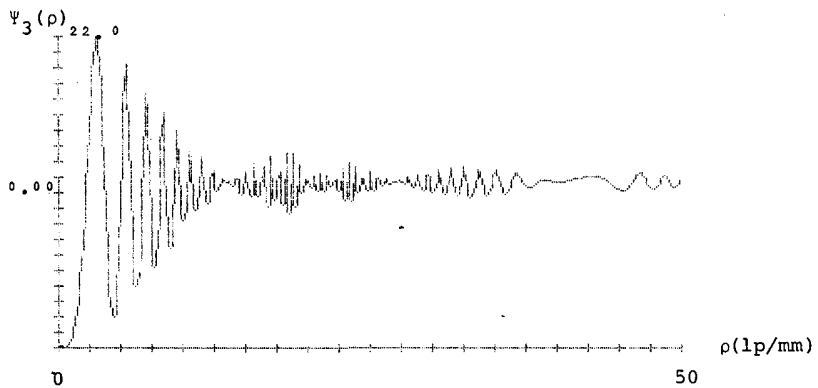

Fig. 9. Cellular membrane contribution to the total diffracted complex amplitude distribution for the real transmission function. 


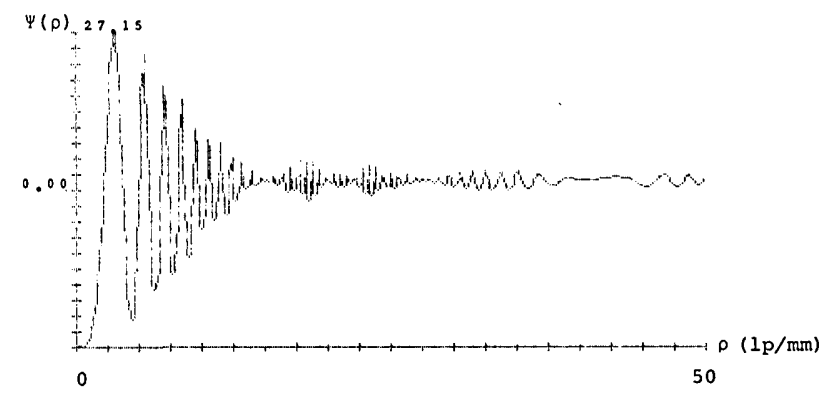

Fig. 10. Total diffracted amplitude distribution for the real transmission function.

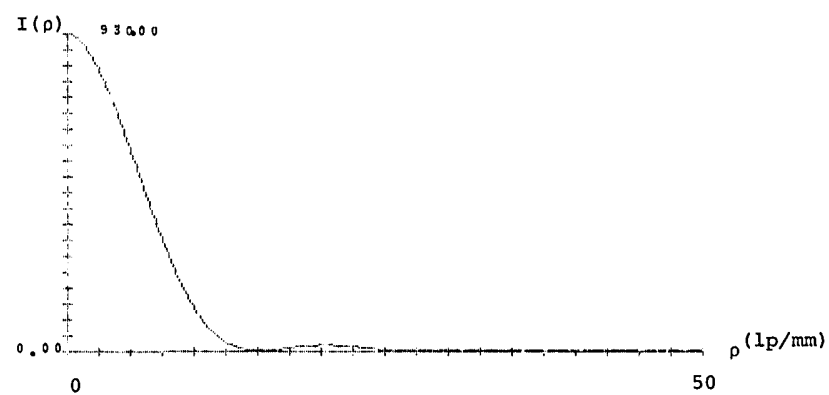

Fig. 11. Total diffracted intensity distribution for the real transmission function.

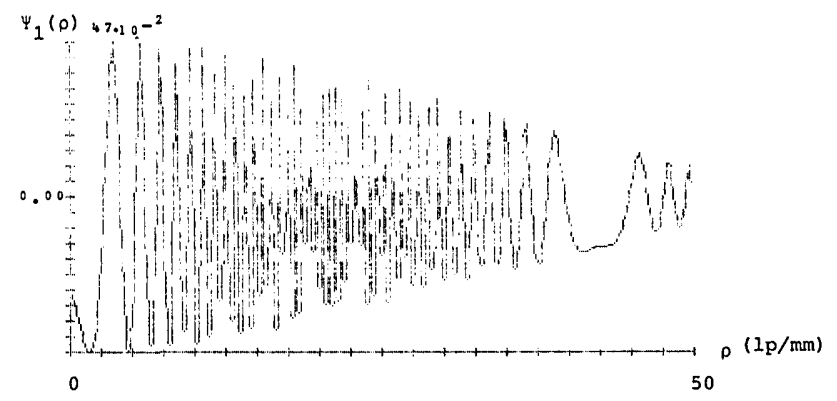

Fig. 12. Nucleus contribution to the total diffracted complex amplitude distribution for the pure phase transmission function $\left(\Phi_{N}=\right.$ $\left.\pi / 2, \quad \Phi_{C}=0, \quad \Phi_{M}=\pi / 2\right)$.

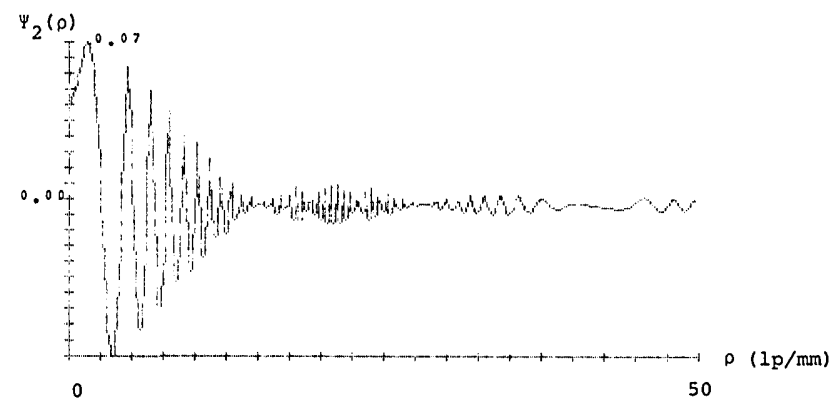

Fig. 13. Cytoplasm contribution to the total diffracted complex amplitude distribution for the pure phase transmission function $\left(\Phi_{N}\right.$ $\left.=\pi / 2, \quad \Phi_{C}=0, \quad \Phi_{M}=\pi / 2\right)$.

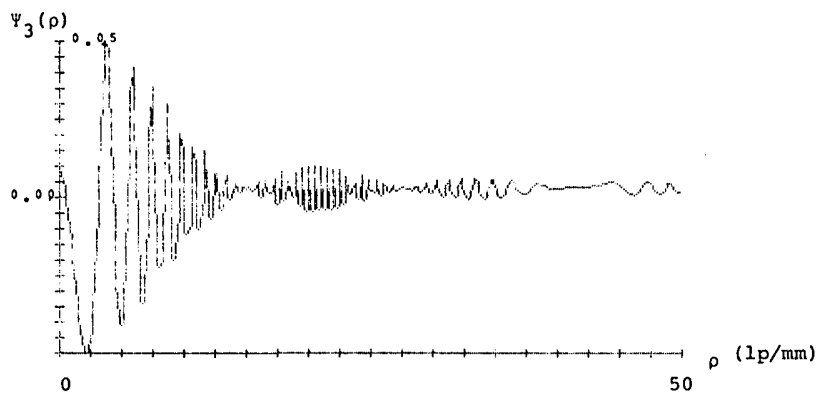

Fig. 14. Membrane contribution to the total diffracted complex amplitude distribution for the pure phase transmission function $\left(\Phi_{N}\right.$ $\left.=\pi / 2, \quad \Phi_{C}=0, \quad \Phi_{M}=\pi / 2\right)$.

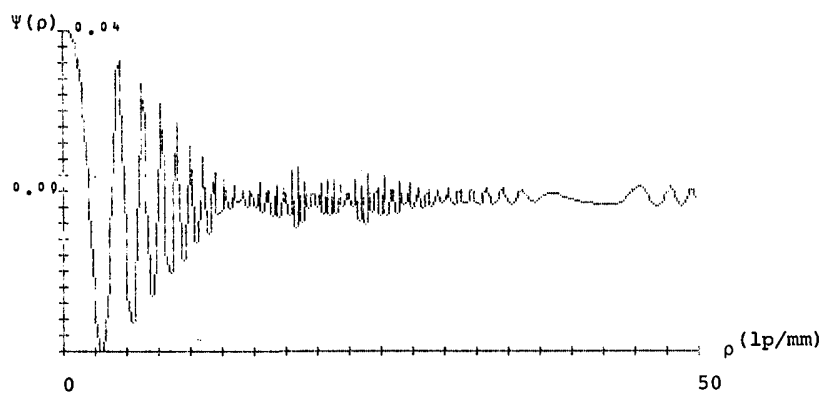

Fig. 15. Total diffracted amplitude distribution for the pure phase transmission function $\left(\Phi_{N}=\pi / 2, \quad \Phi_{C}=0, \quad \Phi_{M}=\pi / 2\right)$.

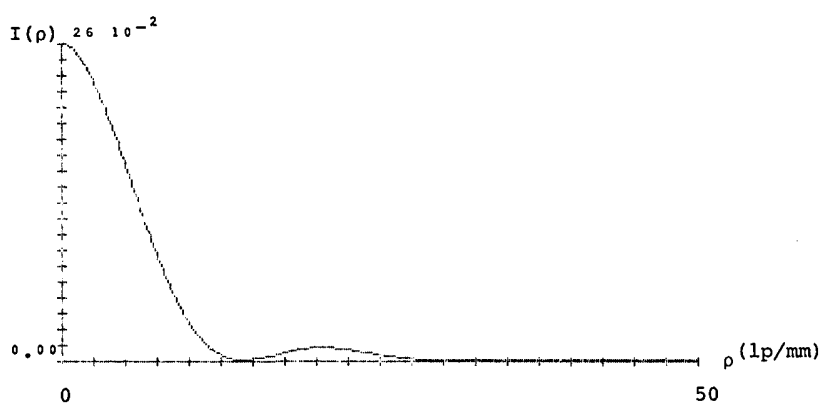

Fig. 16. Total diffracted intensity distribution for the pure phase transmission function $\left(\Phi_{N}=\pi / 2, \quad \Phi_{C}=0, \quad \Phi_{M}=\pi / 2\right)$.

comes from the membrane region; both total amplitude and intensity distributions appear in Figs. 10 and 11. In this case the second secondary maximum present in Fig. 6 disappears.

\section{Numberical Results for the Pure Phase Transmission Function}

As in the general case we studied separately the contribution to the total complex diffracted amplitude distribution for the nucleus, the cytoplasm, and the cellular membrane, and we varied the phase values from $\Phi=0$ to $\Phi=\pi / 2$ and from $\Phi=\pi$ to $\Phi=3 \pi / 2$. The maximum contribution takes place for the following phases: $\Phi_{N}=\pi / 2, \Phi_{C}=\pi / 2$, and $\Phi_{M}=\pi / 2$. These 
results do not have a relevant physical meaning as the three regions of the cell considered present the same phase term. Then, we considered the second maximum value which takes place for $\Phi_{N}=\pi / 2, \Phi_{C}=0$, and $\Phi_{M}$ $=\pi / 2$. That is, the phase terms for the nucleus and the membrane are maxima, while the cytoplasm has a vanishing contribution in the phase. The results obtained are displayed in Figs. 12-14 for the nucleus, the cytoplasm, and the cellular membrane, respectively. The total diffracted amplitude and intensity distributions are shown in Figs. 15 and 16 . In the latter, only a secondary maximum appears as in the real transmission function case, although it has a higher intensity. This fact supposes a loss in the information contained in the diffraction pattern with respect to the general case.

\section{Conclusions}

Several conclusions can be arrived at from the results presented above:

(1) If a real transmission function for the biological cell is assumed, there is a strong contribution from the membrane transmission function.

(2) If we assume that the attenuation coefficient = 1 , the pure phase transmission function is obtained. It is then found that the three separate partial contributions coming from the nucleus, the cytoplasm, and the cellular membrane oscillate strongly and it is possible to define three different profiles in the oscillations of the three mentioned regions. Note that the positions of the pulses depend on the spatial frequency values of the diffraction pattern. In the present study the values are assumed to be extended to 50 lines $/ \mathrm{mm}$. The maximum contribution to the total diffracted amplitude distribution corresponds to the membrane zone.

(3) If the transmission function contains real and complex terms, the most important contribution is from the membrane region which clearly predominates over the total amplitude response.

(4) As a resume of the three above conclusions we can assume that none of the three regions of a biological cell can be neglected if Fourier analysis s used to obtain information from its structure. Considering a parabolic variation for the membrane produces an increase in the information contained in the cellular membrane.

This work was presented at the Tenth IOCC-ICO Congress, Cambridge, Mass., April 1983. It was partially financially supported by the Spanish Advisory Commission for Scientific and Technical Research under grant 4219/79.

\section{References}

1. M. Kerker, The Scattering of Light and other Electromagnetic Radiation (Academic, New York, 1969).

2. A. Ishimaru, Wave Propagation and Scattering in Random Media (Academic, New York, 1978).

3. R. A. Meyer, Appl. Opt. 16, 2036 (1977).

4. N. Catsimpoolas, Ed., Cell Analysis, Vol. 1 (Plenum, New York, 1982).

5. J. W. Goodman, Introduction to Fourier Optics (McGraw-Hill, New York, 1968).

\section{Positions Open In NSF}

Applicants for the following positions should submit resumes including current salary to NSF Personnel Administration Branch, Room 212, 1800 G St., NW, Washington, DC 20550. Attn: E. Paul Broglio (357-7840). Women, minorities, and handicapped scientists are encouraged to apply. NSF is an Equal Opportunity Employer.

- NSF's Division of Mathematical and Computer Sciences is seeking qualified applicants for positions which periodically become available. The positions will be filled on a one- or two-year rotational basis and are excepted from the competitive civil service. The salary ranges from: $\$ 30,000$ to $\$ 45,000$ for assistant program director; $\$ 35,000$ to $\$ 55,000$ for associate program director; and, $\$ 40,000$ to $\$ 65,000$ (currently limited by statute to $\$ 63,800$ ) for program director. Applicants should have a Ph.D. or equivalent experience and training in an appropriate field. In addition, applicants should have successful scientific research experience beyond the Ph.D. as follows: three to four years for the assistant program director; four to six years for the associate program director; and six to eight years for the program director. A broad, general knowledge of computer research and some administrative experience are also required.

- NSF's Division of Chemistry is seeking qualified applicants for program director and associate program director positions in structural chemistry, thermodynamics, dynamics, chemical physics, chemical analysis, and chemical instrumentation for 1984-85. The salary ranges from $\$ 40,000$ to $\$ 65,000$ per annum (currently limited by statute to $\$ 03,800$ ) for program director; and $\$ 35,000$ to $\$ 55,000$ for associate program director. Applicants should have a $\mathrm{Ph} . \mathrm{D}$. or equivalent research experience in the area of chemistry related to the program. Recent scientific research experience beyond the Ph.D. are pre-requisites for consideration as follows: four to six years for associate program director and six to eight years for program director.

- NSF's Division of Biotic Systems and Resources is seeking qualified applicants for four positions: program director for Ecosystem Studies, program director for Ecology, program director for Systematic Biology and associate program director for the Systematic Biology program. The positions will be filled on a one- or two-year rotational basis and are excepted from the competitive civil service. The salaries range from: $\$ 40,000$ to $\$ 65,000$ (currently limited by statute to $\$ 03,800$ ) for program director and $\$ 35,000$ to $\$ 55,000$ for associate program director. Applicants should have a Ih.D. and/or equivalent research experience in a related discipline. In addition, applicants should have successful scientific research experience beyond the Ph.D. as follows: four to six years for the associate program director; six to eight years for the program director. 